\title{
Adverse outcomes after percutaneous dilatational tracheostomy versus surgical tracheostomy in intensive care patients: case series and literature review
}

This article was published in the following Dove Press journal:

Therapeutics and Clinical Risk Management

7 August 2017

Number of times this article has been viewed

\author{
Konrad Jarosz' \\ Bartosz Kubisa ${ }^{2}$ \\ Agata Andrzejewska ${ }^{3}$ \\ Katarzyna Mrówczyńska ${ }^{3}$ \\ Zbigniew Hamerlak ${ }^{4}$ \\ Alicja Bartkowska- \\ Śniatkowska ${ }^{5}$ \\ 'Department of Clinical Nursing, \\ Pomeranian Medical University, \\ ${ }^{2}$ Thoracic Surgery and Transplantation \\ Department, Pomeranian Medical \\ University, ${ }^{3}$ Anaesthesiology \\ and Intensive Care Department, \\ Pomeranian Medical University, \\ ${ }^{4}$ Stomatology Department, \\ Pomeranian Medical University, \\ ${ }^{5}$ Anaesthesiology and Pediatric \\ Intensive Care Department, Poznan \\ University of Medical Sciences, \\ Szczecin, Poland
}

\begin{abstract}
Tracheostomy is a routinely done procedure in the setting of intensive care unit (ICU) in patients requiring prolonged mechanical ventilation. There are two ways of making a tracheostomy: an open surgical tracheostomy and percutaneous dilatational tracheostomy. Percutaneous dilatational tracheostomy is associated with fewer complications than open tracheostomy. In this study, we would like to compare both techniques of performing a tracheostomy in ICU patients and to present possible complications, methods of diagnosing and treating and minimizing their risk.
\end{abstract}

Keywords: tracheostomy, percutaneous tracheostomy, percutaneous dilatational tracheostomy, bronchoscopy, surgical tracheostomy, tracheoesophageal fistula, tracheostomy complications

\section{Introduction}

Nowadays, tracheostomy is a common surgical procedure performed in critically ill patients in the intensive care unit (ICU). ${ }^{1}$ Emergency situations like "can't intubate, can't ventilate" used to be the main indications to perform a surgical intervention to open the obstructed airway. Notwithstanding, tracheostomy is mostly performed as an elective procedure. The need for prolonged intubation for long-term mechanical ventilation and a failure to wean from the ventilator in a critically ill patient is the main indication to perform a tracheostomy. It is also performed to ease tracheobronchial toilet, lowering the risk of ventilator-associated pneumonia (VAP), and reduce the dose of sedative drugs. Other indications include facial trauma, severe injury to the neck or larynx, patients with severe obstructive sleep apnea intolerant of continuous positive airway pressure therapy or as a preemptive therapy in oncological or reconstructive surgery of head or neck. Failure to extubate the patient depends on many factors such as chronic lung disease, vocal cord palsy and so on. ${ }^{2,3}$ Possible difficulties with intubation due to edema, tumor on the face or neck or other known anatomical abnormalities and dysmorphies may be an indication to perform a tracheostomy. ${ }^{4}$ Considering the abovementioned indications, there are no absolute contraindications to perform this procedure. Relative contraindications include coagulopathy, difficult anatomy (eg, tracheal deviation and vascular abnormalities), narrowing or obstruction in bronchial tree, recent cervical spine surgery or unstable fractures of cervical spine and local neck infection. ${ }^{5}$ What is more interesting is that the indications for both surgical and percutaneous dilatational tracheostomy are identical. Relative contraindications for percutaneous
Correspondence: Agata Andrzejewska Anaesthesiology and Intensive Care Department, Pomeranian Medical University, Unii Lubelskiej I, 7I-252

Szczecin, Poland

Tel +48605673463

Email november.rain@wp.pl 
dilatational tracheostomy (PDT) are small children, patients with short thick neck, anatomical abnormalities or high bifurcation of the brachiocephalic trunk. ${ }^{4}$ Throughout the years, tracheostomy technique evolved from surgical open tracheostomy, but thanks to the evolution of equipment and doctors skills, tracheotomy has became a less invasive procedure which can be simply done at the bedside. ${ }^{6}$ The number of performed percutaneous tracheostomies in the ICU is still increasing. There are a number of different techniques of performing a PDT divided into single- or multiple-step techniques or a retrograde tracheostomy. Most popular singlestep PDTs are Griggs and Schachner techniques, whereas multiple-step techniques are Ciglia and Fanconi. Single-step techniques are associated with higher success rate and fewer minor complications, whereas retrograde tracheostomy is considered to be the least effective and with the highest risk of conversion to the open technique. ${ }^{3}$ Bronchoscopic guidance is recommended every time PDT is performed. It significantly lowers the risk of complications; however, it is still not routinely used in many European centers. ${ }^{7-9}$ In our hospital, most of the percutaneous tracheostomies are done with Griggs technique under bronchoscopic guidance. Bronchofiberoscopy helps us to identify puncture site on the anterior wall of the trachea and how deep the cannula is inserted which helps us to prevent puncture of the posterior wall of the trachea.

The most common complications of tracheostomy, irrespective of the technique that is used, are loss of airway, hemorrhage, obstruction of tracheostomy, tube misplacement, unintended decannulation, laceration of the posterior tracheal wall, pneumothorax, subcutaneous emphysema and breakage of the tracheal cartilages. The most frequent late complications are stomal infection, subcutaneous and/or mediastinal abscess, tracheoesophageal fistula and subglottic tracheal stenosis. ${ }^{10}$ One of the most feared of and not a very rare complication is placement of the cannula between mucosa and tracheal wall resulting with a false passage. It can be a very serious complication if the diagnosis is made too late. Tracheostomy tube displacement, especially in the immediate period following tracheostomy when there is still lack of formal stoma creation and a narrower tracheocutaneous tract, is a life-threatening complication. We can divide tracheostomy complications into three groups depending on the timing of their occurrence: immediate, early and late (Table 1$){ }^{3}$

Early complications can be diagnosed and treated almost immediately, for example hemorrhage can be controlled by putting some pressure on the place of bleeding or with surgical intervention. Stomal site infections can be treated with targeted antibiotic therapy or with alternative
Table I Tracheostomy complications

\begin{tabular}{lll}
\hline Immediate & Early & Late \\
\hline - Hemorrhage & - Bleeding & - Tracheal stenosis \\
- Damage to & - Cannula displacement & - Granuloma \\
the tracheal & - Pneumothorax & - Tracheomalacia \\
structures & - Pneumomediastinum & - Pneumonia \\
- Failure & - Subcutaneous & - Aspiration \\
- Aspiration & emphysema & - Tracheoartierial \\
- Air embolus & - Stomal infection & fistula \\
- Loss of the & - Necrotizing stomal & - Tracheoesophageal \\
airway & infection & fistula \\
- Hypoxemia, & - Unintended & - Unintended \\
hypercarbia & decannulation & decannulation \\
- Death & - Dysphagia & Dysphagia \\
\hline
\end{tabular}

local treatment. Early diagnosis and adequate treatment significantly reduce the duration of the therapy and may prevent deterioration of the overall condition of the patient. Other, less specific symptoms such as dysphagia, excessive secretion, tracheotomy tube intolerance or recurrent pneumonias should be carefully monitored and at the time of diagnosis appropriate treatment should be given immediately. Late complications, especially when the time of diagnosis is delayed, usually require more complex therapy. At this time, key to a successful treatment is a multidisciplinary approach, which helps to choose the most appropriate treatment, such as excision of the granuloma or stenting and protection of the trachea. Timing of the diagnosis and appropriate treatment for the patient are the most important when it comes to dealing with complications no matter whether they are immediate, early or late.

In this study, we present two case reports, regarding percutaneous and surgical tracheostomy (ST) and their complications: tracheoesophageal fistula and a major infection of postoperative wound, respectively. Written informed consent has been obtained from the patients for the publication of their case details and any accompanying images.

\section{Case no I}

A 59-year-old patient was transferred from Neurology Unit to the ICU due to respiratory failure after hemorrhagic stroke and left-sided hemiparesis. Past medical history of the patient revealed atrial fibrillation, arterial hypertension, dyslipidemia and obesity. At the time of admission in ICU, patient was in critical condition requiring infusion of catecholamines and mechanical ventilation.

With expected prolonged mechanical ventilation, after 11 days in the ICU, we performed an elective PDT to our patient. Procedure was performed with Griggs technique under bronchofiberoptic guidance. During the procedure, 
visual track of the bronchofiberoscope failed. The incision in the anterior trachea wall was already properly made, so we decided to continue without direct visual guidance. Insertion of the metal guide wire and as a result tracheostomy tube positioning could not be confirmed. Despite the proper incision in the trachea, the cannula could not be placed. Patient was intubated again. Subcutaneous emphysema on the neck and upper part of the chest developed shortly after the procedure. We qualified the patient for surgical revision in the operating room by a laryngologist. No significant damage to the trachea was noticed during tracheal wound inspection. During this whole procedure and open ST, no ventilation problems were reported. Few days after surgery, patient was successfully off the ventilator, breathing through the tracheostomy tube. Throughout the patients stay in the ICU, we noticed occasional leakage of chyme from around the tracheostomy and this continued even after the cuff was sealed. Ten days after the surgery, in the operating room because of the previous difficulties with tracheostomy tube exchange and need for retracheostomy, the tube was changed, without any complications. Few days later, the patient underwent percutaneous endoscopic gastrostomy (PEG) in the Gastroenterology Unit. Surprisingly, during the endoscopic procedure, tracheostomy tube cuff was found in the esophagus, which suggested tracheaesophageal fistula. It was confirmed on CT scan. The CT scans showed the cuff of the tube moves from the trachea to the esophagus (Figure 1). We qualified the patient for treatment in thoracic surgery unit, including tracheal stenting. Awaiting surgical intervention, we changed patient's tracheostomy tube for the

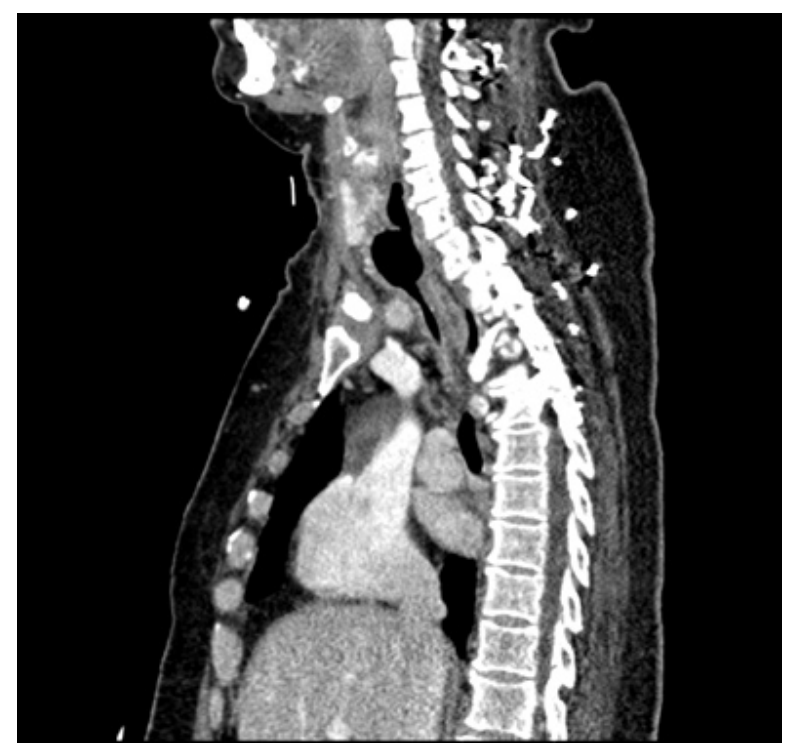

Figure I Computed tomography scan.

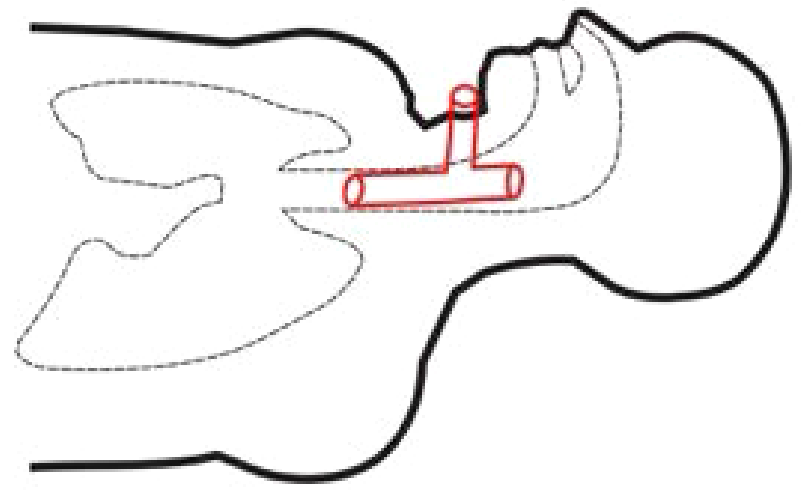

Figure 2 Montgomery tube placement.

one with integrated suction feature and she stayed on total parenteral nutrition.

During the procedure of tracheal stenting, bronchofiberoscope was inserted into the trachea, the tracheostomy tube was then removed and next, with help of fiberoptic forceps, an umbilical tape was inserted. Later on, surgeon placed the Montgomery tube inside the trachea and trimmed it with the umbilical tape. The T-tube was fixed and its positioning was checked, with the distal limb $2 \mathrm{~cm}$ above tracheal bifurcation and proximal limb under the vocal cords. The extraluminal limb was protruding through tracheotomy stoma. Finally, the esophagus checked endoscopically to make sure the stent has not moved from trachea to esophagus - just linear mucosal damage $3 \mathrm{~cm}$ long at the proximal $1 / 3$ of the esophagus, which corresponded with a localization of the diagnosed before fistula. No complications throughout this period were reported. Figures 2 and 3

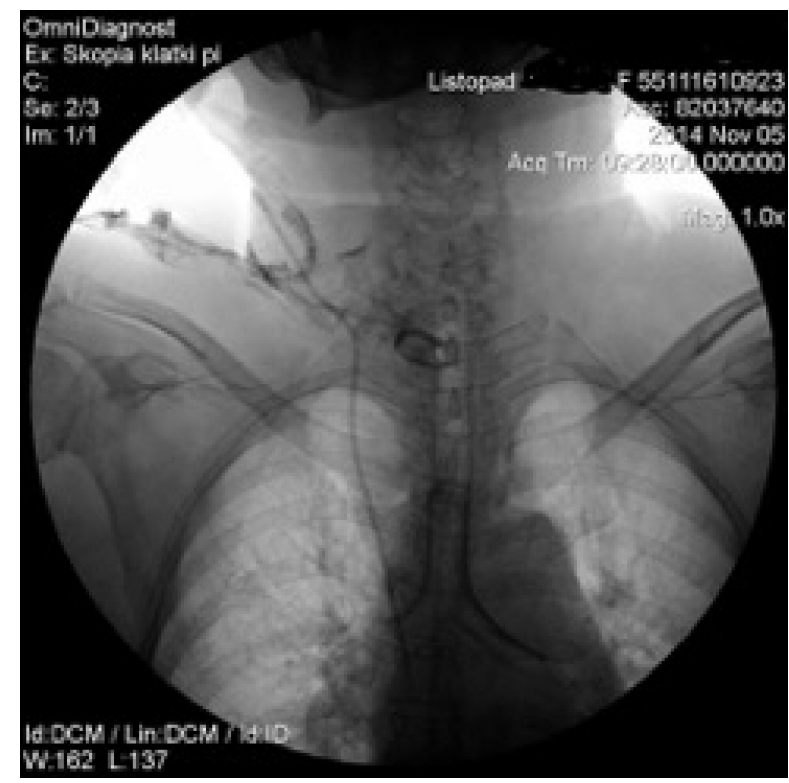

Figure 3 Chest $X$-ray after the procedure. 
are graphic and radiologic presentation of this procedure. Two days after the stenting procedure, patient was readmitted to the Neurology Unit. After the Montgomery tube was placed, no chyme leakage from around the tracheostomy was observed. From the Neurology Unit, patient was transferred to the long-term neurorehabilitation facility.

\section{Case no 2}

A 21-year-old male with Duchenne muscular dystrophy (diagnosed and confirmed genetically while the patient was 4 years old), massive scoliosis, tetraplegia, cachexia and epilepsy on long-term noninvasive mechanical ventilation due to chronic pulmonary failure was qualified by general medicine specialist to tracheostomy and PEG. To perform both these procedures, patient had a scheduled admission to the ICU. Instead of usually done PDT under bronchofiberoptic guidance, we decided to perform an open ST in cooperation with laryngologists, due to anatomical abnormalities in patient's trachea (trachea was rotated to the left, with palpable membranous part on the front of the neck). In the operating room under general anesthesia, X-shaped cut was made between cricoid cartilage and suprasternal notch. Prelaryngeal tissues and isthmus were separated and moved upward revealing oblique positioning of the trachea relative to median line of the body. Vertical incision on the 3 and 4 cartilage was made and horizontal incision was made on the membrane between them. Tracheoplasty was performed. Five days after the surgery, because of the wound dehiscence, patient required revision of the tracheostomy and retracheoplasty in the setting of operating room. Skin sutures were removed, revealing the tracheocutaneous tract which was oblique and turned to the right tissues on the left pole of the stoma were dehisced. Laryngologist performed surgical debridement and retracheoplasty. Finally, skin and cartilages were anastomosed with insoluble sutures. Next step of the treatment scheduled for the patient was PEG done in Gastroenterology Department. There were no problems with passing through the esophagus with endoscope, and the procedure was successful. During the hospitalization, there was a late complication - local infection of the skin around the tracheostomy and an extensive necrosis causing the tracheotomy tube displacement. This complication required long-term, intensive treatment with not only systemic antibiotic therapy but also local treatment including laser therapy and ointments customized for the patient. Ointment formula was made by a doctor specialized in treatment of difficult wound infections. Laser therapy included laser BTL-4110

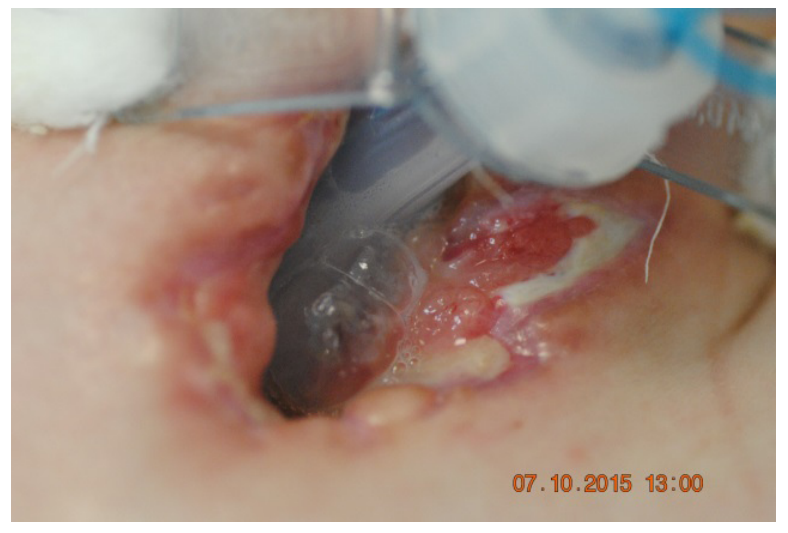

Figure 4 Before treatment.

Topline (Industrial Ltd, Stevenage Hetfordshire, UK) with multibeam laser probe with 18 diodes and radiation: red $685 \mathrm{~nm} / 30-50 \mathrm{~mW}$, infrared $830 \mathrm{~nm} / 100-400 \mathrm{~mW}$ and combined until 1,800 mW. Treatment consisted of 9 sessions each lasting 2 minutes, focused on the wound area. Topical treatment included customized ointment composed of $85 \mathrm{~g}$ allantoin ointment 2 packages, panthenol spray $1 / 5$ p, solcoseryl dental $5 \mathrm{~g}$, dexaven dental $5 \mathrm{~g}$ and metronidazole dental $5 \mathrm{~g}$. It was a dose for 1 month treatment. Dressings were changed daily. Complementary treatment included Kinesiology taping (Nittodenko, Osaka Prefecture, Japan). Local treatment lasted 35 days with good results, and much improvement was observed during the course of the therapy. When the local effect was satisfactory and the wound almost completely healed, we discharged the patient out of the hospital and forwarded him to palliative care with home ventilator program. Local treatment with Kinesiology Taping and ointments was continued for next 4 months, with very good final - total wound healing and proper formal stoma and tracheocutaneous tract creation (Figures 4 and 5 present wound before and after the treatment).

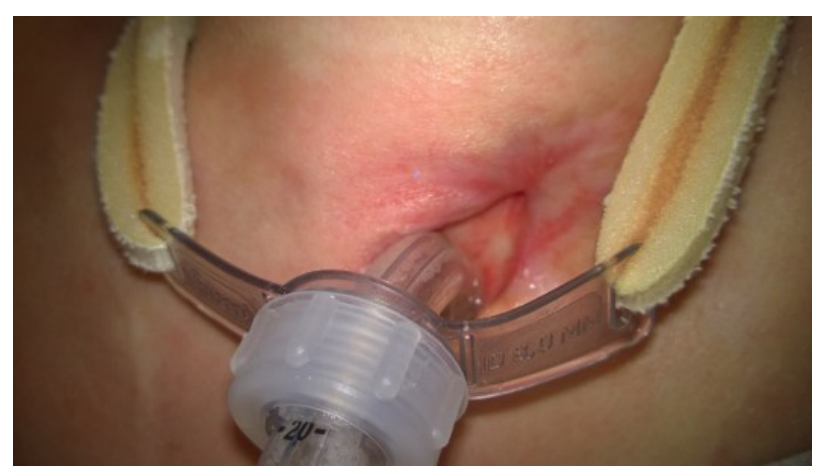

Figure 5 After local treatment. 


\section{Discussion}

Both the clinical cases that we presented in this study refer to possible complications of tracheostomy and show alternative ways of treatment. In case no 1, we described a complication of a PDT - a tracheoesophageal fistula. The second case refers to ST complication - an extensive wound infection, requiring local and systemic treatment. Tracheoesophagal fistula is a rare complication associated with perforation of a posterior tracheal wall made during the procedure or a late complication secondary to excessive cuff pressures. Treatment of choice is surgical closing of the fistula or stenting of the trachea. ${ }^{11}$ To date, there are not many reports concerning the incidence of tracheoesophageal fistulas during and after the PDT and this is considered as a very rare complication. Our patient was treated with a Montgomery tube, which was introduced in the mid-1960s to support the trachea following laryngotracheoplasty. In its original form, the device is an uncuffed silicone T-tube that is inserted with the long limb in the trachea and the short limb projecting through the tracheostomy stoma. The short limb can be closed allowing the patient to normally breathe through the larynx and allows proper phonation. ${ }^{12}$ Nowadays, T-tubes are mostly used in benign tracheal diseases such as tracheomalacia, sarcoidosis, caustic injury to the trachea due to ingestion or inhalation of chemical irritants or as a palliative measure in malignant tracheal diseases including primary tracheal tumors, esophageal cancers with involvement of the trachea and metastatic cancer to the trachea. It can also be used as a temporary measure in patients with a temporary contraindication to the surgery or in freshly injured patients who needed immediate airway support. Montgomery tubes are well tolerated, and there are even some reports about patients who have had T-tubes for over a 20-year period. Benign tracheal diseases, such as tracheal stenosis after ST, are mostly related to the use of artificial airways or are an iatrogenic complication. ${ }^{13,14}$ One of the indications for the T-tube is tracheoesophagal fistula, a complication after tracheostomy or due to prolonged intubation. Montgomery tubes rarely cause serious complications. Complications include subcutaneous emphysema following insertion, migration requiring removal and reinsertion, posterior displacement into the trachea causing acute airway obstruction, buildup of dried luminal respiratory secretions needing bronchoscopic suctioning or removal of T-tube, airway infection, tracheal hemorrhage, formation of granulation tissue and prolonged healing of tracheocutaneous fistula. When the proximal end of the T-tube is positioned just above the vocal cords to stent the subglottic area, additional complications are encountered, including aspiration and voice weakening. ${ }^{14}$

The most common complication of PDT is bleeding, which can be stopped by local pressure (31.7\%), and perforation of the endotracheal tube cuff during the procedure $(20.2 \%) .{ }^{7}$ It is recommended to use a bronchofiberoptic guidance during the PDT which increases safety of the procedure and significantly decreases the risk of complications, such as perforation of posterior tracheal wall or tracheostomy tube misplacement. ${ }^{3,8,9}$ Performing a PDT without a routine bronchoscopy is still considered to be a safe procedure, but as our case shows that loss of visual guidance during the PDT can result in a serious complication requiring a complex and invasive treatment. Bronchofiberoscopy has become a very common diagnostic and/or therapeutic tool in ICU. ${ }^{15}$ It is considered to be a very safe procedure, and possible benefits exceed an overall risk of complications. Furthermore, during bronchofiberoscopy, microbiological material can be directly sampled from the respiratory tract, ${ }^{16,17}$ biopsy sample can be taken and source of bleeding identified, what can help to achieve a diagnosis and start the right and immediate treatment. Bronchofiberoscopy seems to be also useful for the treatment of atelectases and removing of foreign body or residual mucous so that the collapsed lung can expand. ${ }^{16,18,19}$

Nowadays, ST has been rarely done as the PDT becomes a method of choice in the ICU patients. In studies comparing PDT and ST, the overall risk of complications is similar, but the risk of complications such as stomal infection or bleeding is higher in surgical technique. ${ }^{20}$ In a trial with more than 3000 PDTs without routinely performed bronchoscopy, major airway complications occurred in $0.38 \%$ patients, accounting for $0.16 \%$ deaths. Early major complications included 3 airway losses and 1 bleeding event requiring formal exploration with procedure-related deaths occurring in 3 patients. Intermediate major complications included 2 tube occlusion/ dislodgement events with 2 related deaths. Late complications included $5(0.16 \%)$ cases of tracheal stenosis requiring intervention without associated deaths. ${ }^{21}$ Tracheal stenosis as a late complication also appeared more often in patients who had ST. ${ }^{22}$ There is no significant difference in hemorrhage incidence and mortality when PDT and ST were compared. However, lower risk of infection and shorter duration of the procedure favors the percutaneous technique. In addition, PDT is cost effective and decreases the risk associated with patient transport to and from the operating room, what is especially important with patients in critical condition and those with bed regime., $3,22,23$ 
When we decide to perform a tracheostomy on a patient, we should take into consideration all the possible advantages of a tracheostomy such as shorter ICU stay, shorter duration of mechanical ventilation, less sedative drugs and easier tracheobronchial toilet and fewer VAP and lower mortality rate. ${ }^{24}$ However, we must keep in mind that this is an invasive procedure and should be aware of the possible complications. Timing of tracheostomy in ICU patients is still a matter of discussion and there is still no universal consensus on when to perform tracheostomy. However, there are a lot of data supporting early tracheostomy, but on the other there are some new data showing no advantages of performing tracheostomy within 4 days of admission to ICU. Recently published results from the TracMan trial showed no improvement in early tracheostomized patients in 30-day mortality, mortality in critical care, length of stay in critical care and antimicrobial-free days. However, there was a reduction in the sedative use in the early tracheostomy group. ${ }^{25}$ Expected length of mechanical ventilator support is the most important consideration when it comes to the timing of the tracheostomy. $3,5,26$

\section{Conclusion}

Tracheostomy is one of the most common ICU procedures performed. PDT under bronchofiberoptic guidance is a safe and effective procedure. PDT should become a method of choice in ICU patients. Serious complications such as laceration of the posterior tracheal wall or tracheoesophageal fistula are very rare. However, it is very important that they are diagnosed and treated very fast. To avoid such complications, it is recommended to use bronchofiberoptic guidance during the percutaneous dilatational tracheostomy. One of the therapeutic options for this type of complication could be Montgomery tube, which preserves normal respiration while the fistula heals. Patients with relative contraindications to PDT, such as anatomical abnormalities, should have thorough radiological end endoscopic check-up to verify them and not to give up on PDT too quickly. When ST is necessary, it is associated with higher risk of stomal infection, where sometimes laser therapy and/or medication of above proposed composition can be the only effective treatment.

Tracheostomy, despite being a rather safe procedure, still carries some risk of complications. Early diagnosis and proper treatment of them can significantly lower mortality rate in patients undergoing such procedures.

\section{Disclosure}

The authors report no conflicts of interest in this work.

\section{References}

1. Korpas-Wasiak M, Śmiechowicz K, Machała W, Lesiak P. Tracheotomia w oddziałach intensywnej terapii. Anestezjologia i Ratownictwo. 2014;8:181-188.

2. Blot $\mathrm{F}$, Melot $\mathrm{CH}$. Indications, timing and techniques of tracheostomy in 152 French ICUs. Chest. 2005;127(4):1347-1352.

3. Cheung NH, Napolitano LM. Tracheostomy: epidemiology, indications, timning, technique and outcomes. Respiratory Care. 2014; 59(6):895-915.

4. Patel SA, Meyer TK. Surgical airway. Int J Crit Illn Inj Sci. 2014;4(1): 71-76.

5. Cipriano A, Mao ML, Hon HH, et al. An overview of complications associated with open and percutaneous tracheostomy procedures. Int J Crit Illn Inj Sci. 2015;5(3):179-188.

6. Polderman KH, Spijkstra JJ, de Bree R, et al. Percutaneous dilatational tracheostomy in the ICU: optimal organization, low complication rates, and description of a new complication. Chest. 2003;123(5):1595-1602.

7. Vargas M, Sutherasan Y, Antonelli M, et al. Tracheostomy procedures in the intensive care unit: an international survey. Crit Care. 2015; 19:291.

8. Brunetti I, Pelosi P. Ultrasound and bronchoscopic guided percutaneous dilatational tracheostomy: friends for life! Minerva Anestesiol. 2015;81(2):119-121.

9. Saritas A, Saritas PU, Kurnaz MM, Beyaz SG, Ergonenc T. The role of fiberoptic bronchoscopy monitoring during percutaneous dilatational tracheostomy and its routine use into tracheotomy practice. $J$ Pak Med Assoc. 2016;66(1):839.

10. Roxbury C, Qualliotine J, Molena D, Kim Y. Unusual airway complication after percutaneous tracheotomy: case report and literature review. Laryngoscope. 2015;125(8):1883-1885.

11. Li Z, Zhong CH. Montgomery T-tube for the management of postintubation tracheoesophageal fistula with synchronic subglottic laryngotracheal stenosis. Thorac Cardiovasc Surg Rep. 2014;3(1): 61-63.

12. Guha A, Mostafa SM, Kendall JB. The Montgomery T-tube: anaesthetic problems and solutions. Br J Anaesth. 2001;87(5):787-790.

13. Carretta A, Casiraghi M, Melloni G, et al. Montgomery T-tube placement in the treatment of benign tracheal lesions. Eur J Cardiothorac Surg. 2009;36(2):352-356.

14. Momen M. Wahidi MD, Armin Ernst MD. The Montgomery T-tube tracheal stent. Clin Chest Med. 2003;24(3)437-443.

15. British Thoracic Society Bronchoscopy Guidelines Committee, a Subcommittee of the Standards of Care Committee of the British Thoracic Society. British Thoracic Society guidelines on diagnostic flexible bronchoscopy. Thorax. 2001;56:(Suppl 1):S1-S21.

16. Verma A, Sim WY, Tai DY, et al. Role of bronchoscopy in prompt discharge from the intensive care unit. J Bronchology Interv Pulmonol. 2016;23(2):123-130.

17. Guidry CHA, Mallicote MU, Petroze RT, et al. Influence of bronchoscopy on the diagnosis of and outcomes from ventilator-associated pneumonia. Surg Infect (Larchmt). 2014;15(5):527-532.

18. Lucena CM, Martínez-Olondris P, Badia JR, et al. Fiberoptic bronchoscopy in a respiratory intensive care unit. Med Intensiva. 2012;36(6): 389-395. Spanish.

19. Kreider ME, Lipson DA. Bronchoscopy for atelectasis in the ICU: a case report and review of the literature. Chest. 2003;124(1): 344-350.

20. Delaney A, Bagshaw SM, Nalos M. Percutaneous dilatational tracheostomy versus surgical tracheostomy in critically ill patients: a systematic review and meta-analysis. Crit Care. 2006;10(2):R55. 
21. Dennis BM, Eckert MJ, Gunter OL, Morris JA Jr, May AK. Safety of bedside percutaneous tracheostomy in the critically ill: evaluation of more than 3,000 procedures. J Am Coll Surg. 2013;216(4):858-865; discussion 865-867.

22. Dempsey GA, Morton B, Hammell C, Williams LT, Tudur Smith C, Jones T. Long-term outcome following tracheostomy in critical care: a systematic review. Crit Care Med. 2016;44(3):617-628.

23. Douglas D, Massick MD, Shonan Yao MD, et al. Bedside tracheostomy in the intensive care unit: a prospective randomized trial comparing open surgical tracheostomy with endoscopically guided percutaneous dilatational tracheotomy. Laryngoscope. 2001;111(3):494-500.
24. Young D, Harrison DA, Cuthbertson BH, Rowan K; TracMan Collaborators. Effect of early vs late tracheostomy placement on survival in patients receiving mechanical ventilation: the TracMan randomized trial. JAMA. 2013;309(20):2121-2129.

25. Combes A, Luyt CE, Nieszkowska A, Trouillet JL, Gibert C, Chastre J. Is tracheostomy associated with better outcomes for patients requiring long-term mechanical ventilation? Crit Care Med. 2007;35(3): 802-807.

26. Puentes W, Jerath A, Djaiani G, Sanchez RC, Wąsowicz M. Early versus late tracheostomy in cardiovascular intensive care patients. Anaesthesiol Intensive Ther. 2016;48(2):89-94.

\section{Publish your work in this journal}

Therapeutics and Clinical Risk Management is an international, peerreviewed journal of clinical therapeutics and risk management, focusing on concise rapid reporting of clinical studies in all therapeutic areas, outcomes, safety, and programs for the effective, safe, and sustained use of medicines. This journal is indexed on PubMed Central, CAS,
EMBase, Scopus and the Elsevier Bibliographic databases. The manuscript management system is completely online and includes a very quick and fair peer-review system, which is all easy to use. Visit http://www.dovepress.com/testimonials.php to read real quotes from published authors.

Submit your manuscript here: http://www.dovepress.com/therapeutics-and-clinical-risk-management-journal 